\section{THE MOVEMENTS OF THE EARTH ${ }^{1}$}

VI.

$W^{E}$ have now to consider some of the results of these Movements of the Earth-first round its own axis, its rotation; then round the sun, its revolution--which we have been considering, results to which of course a general interest attaches, and which there will be no difficulty in showing are of very great importance to us. Occasion was taken to point out that the different appearance presented by the sun and the stars was simply clue to the fact that the sun was very near to us whilst the stars were very distant, the one, a sun which happens to be near to us, the others, also suns, but happening to be very far removed from u1s. Now suppose we have a globe in which we have an electric light, to represent the sun, and a little globe to represent the earth, then it will be obvious that that part of the earth which is turned towards the lamp will be bathed in light, while that half which is turned from it will be in darkness, being, so to speak, only under the light of the distant stars. This shows us the reason for that great difference which we call day and night, and we can quite understand how it is that we get the apparent rise of the sun which occurs when the part of the globe on which we live is carried from the darkness into the light, and sunset which of course occurs when the globe is being carried by its rotation from the light into the darkness. This phenomenon of day and night is thus one of the most obvious results of the rotatory movement of the earth, and one which might have been dismissed in two words had we so chosen, but we will dwell

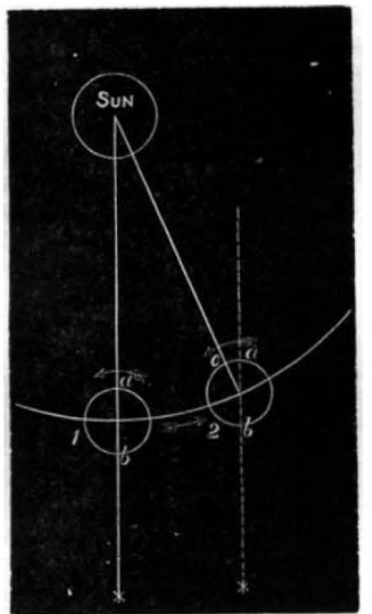

49.-Diagram showing how the difference between the lengths of the sidereal and mean day arises.

upon it fora few moments, because this fundamental difference between day and night furnishes us with a reason why we should discard that sidereal time to which up to the present reference has alone been made.

Fig. 49 will show how it is that under the circumstances in which we thus find ourselves, a new kind of time must take the place of sidereal time. In this diagram we have the earth represented at two positions in its orbit, I and 2 . It travels in this orbit in the direction of the arrows, rotating on its axis the while in the direction also indicated by arrows. Now let us consider the start-point $\mathbf{I}$, and suppose that when the earth occupies this position a particular star is on the meridian at midnight. The earth it will be remembered rotates in twenty-four sidereal hours; it will therefore take twelve hours to turn half round, so that if we consider the sun to be directly opposite the star which is south at midnight it is obvious that they are twelve hours apart. Now consider the earth at position 2. Then remembering this fundamental fact, that the distance of the stars is so enormous that a string stretched from the observer to the star at one point of the earth's orbit would be practically parallel to a string stretched to the same star from any other part of the orbit; it is obvious that the star will have the same right ascension in both positions of the earth, and the line pointing to the star will be practically in the same direction. But the sun will no longer lie along the prolongation of the line joining

$$
1 \text { Continued from p. } 256 .
$$

earth and star as it did at I, for in consequence of the earth's revolution round the sun we shall get a gradually increasing angle as the earth in its orbital course gets farther and farther from its initial position at $\mathbf{I}$. Now it is obvious if we are going to have our time regulated by the sun instead of by the starsand that is what we must do for the purposes of civil life--we shall have to arrange our clock so that when we pass from $\mathbf{I}$ to 2 it must, if it showed I2 o'clock when the sun was due south in the former position, show I 2 o'clock also when the sun is due south in the latter position. If this be so, and we have this angle made by the line joining sun and earth and star, we shall have to make our sun-clock go more slowly than our sidereal clock, for the reason that the sidereal clock will have gone round once in less time than the earth will have got round to the same place with regard to the sun. But if we choose, and we do choose, to say that we will have twenty-four hours from sunsouthing to sun-southing, then these twenty-four hours and necessarily also their minutes and seconds, will be longer than the hours, minutes, and seconds of sidereal time. Let us take another illustration. Consider the case of the earth in three different positions, represented by three globes round a central lamp. Then suppose that in each of these globes a wire is put to represent the direction in which the transit instrument points at Greenwich when the same star is observed at three consecutive intervals of twenty-four hours of sidereal time. These three wires should therefore be placed parallel to each other. Now let us take the electric lamp to represent the sun, then we shall find that, when the transit instrument on each of the earths

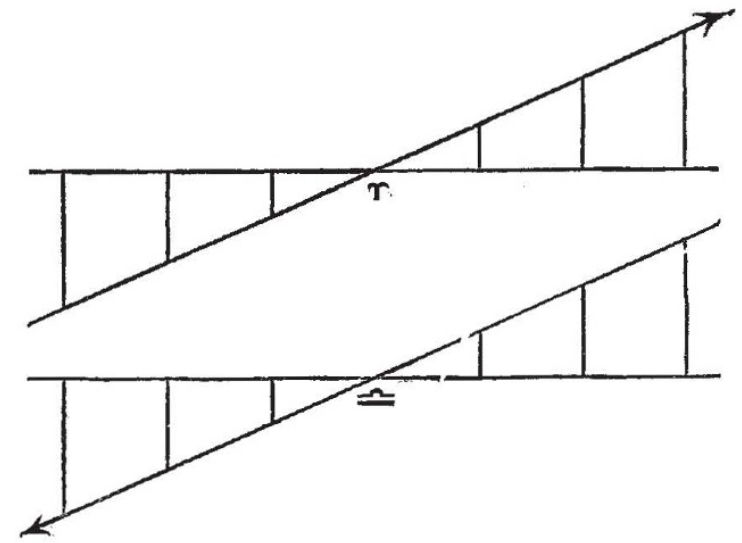

Fig. 50.-Diagram showing how the sun's apparent motion along the inclined lines representing the ecliptic in the direction indicated by the arrow heads is represented by a smaller amount when referred to the earth's equator (the horizontal lines in the figure) at the spring $(\gamma)$ and autumn $(\Omega)$ equinoxes.

is brought round to point at the sun, the three wires which represent the instruments will not be parallel to each other but at some angle. At first sight it might seem that we could easily get a sun-time to replace the star-time, but unfortunately when we go a little deeper into it we find, as we often do in other cases, that it is not quite so easy-and for two reasons. We found, it will be remembered, when we came to consider the form of the earth's orbit, that it was not quite circular, that it was in fact what is called an ellipse, and that the radius vector, i.c: the imaginary line joining the centres of the sun and earth did not sweep through equal arcs in equal times but through equal areas, so that, if we want to invent a clock which will show twenty-four hours from the time of sun-southing one day to the time of sun-southing the next, that clock will require to be regulated differently for every day in the year, because the greater or less part of its orbit moved over by the earth will cause the greater or less angle between the lines joining sun, earth, and star.

That I hope is clear. Thus then there is good reason why this arrangement of having a sun-time from noon to noon will not work. We should have to regulate our clock for every day in the year, or rather for every two opposite days. But there is another matter. We are now in full presence of the fact that the equator of the earth is inclined at an angle of about $23 \frac{1}{2}^{\circ}$ to the plane of the ecliptic. Fig. 50 will perhaps enable us to understand this matter more easily. Let the horizontal lines represent the plane of the equator and the inclined lines the 
plane of the ecliptic. Now our clock and all measurements of time must depend upon the earth's rotation, the plane of which always remains parallcl to itself, and we have seen that our start-point for geocentric and heliocentric longitude depended upon the fact that at a certain point in its revolution the earth passed through a node, and that the node at which the sun with its apparent motion crossed the equator northward was called the ascending node. In the diagram this is represented by $r$ in the upper figure, and the descending node is indicated by $\bumpeq$ in the lower figure. It will be seen that if we have equal intervals along the ecliptic the motion along the equator is represented by bases of successive triangles, of which the hypothenuses lie along the ecliptic. Now the hypothenuse must be

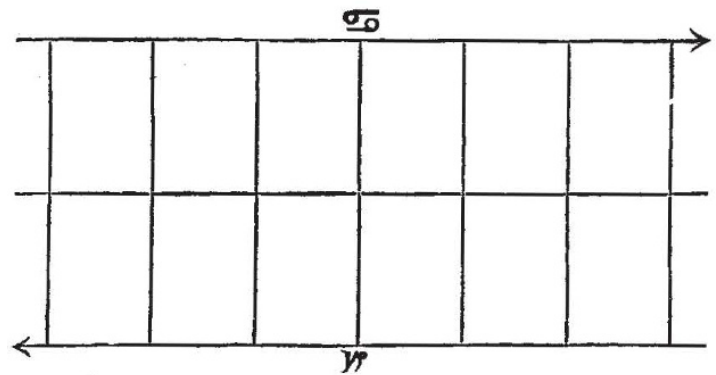

FIG. 5r.-Diagram showing how the sun's apparent motion along the ecliptic, now parallel with the earth's equator (the central line of the figure) at the summer $\left(\sigma_{0}\right)$ and winter $\left(v^{\circ}\right)$ solstices, is represented by equal intervals along the equator.

greater than the base, so that we have at the ascending node the motion of a body along the ecliptic represented only by the base of a triangle of which the motion itself represents the hypothenuse; and the same thing happens in the opposite manner at the descending node; whereas if we take the other positions shown in Fig. 51, for a short time at all events the motion will be parallel, and motion along the ecliptic will be represented by an equal amount along the equator.

These then are the difficulties we have to face when we come to fix our sun-time, first, the unequal velocity of the earth round the sun ; and secondly, those variations which are brought abou by the fact that the two motions of the earth-its axial rotation and yearly revolution-take place in different planes. How are these difficulties got over? They are got over by pretending a sun, as a child would say. Astronomers pretend that there is a sun moving along the equator, or, in other words, they pretend that the earth's movement of revolution takes place in the same plane as its movement of rotation. It is further imagined that this imaginary sun travels at precisely that rate which it would if the average of all its rates along the ecliptic during a year were taken, so that we get something like this (see Fig. 52) first of all we have the curve в в в B, which shows the variation which would take place providing we only had to deal with the obliquity of the ecliptic. Where that curve crosses the horizontal line, we get at those moments (if we disregard the elliptic motion) the same time shown by the mean sun as we should get if the true sun had been taken; it will be seen this occurs four times during the year-on March 20, June 21, September 23, and December 22. Then there is another curve, C C C c, which represents another relation between the mean sun and the true sun. Providing that the two planes were coincident, and that the movement of the earth under these conditions were exactly the same as under the present conditions, namely, that she moved in an ellipse and that the radius vector swept over equal areas in equal times, then we should have the true and mean sun coincident on December $3 \mathbf{I}$ and July $\mathbf{I}$ only. Then the algebraic mean of these two curves, B B B B and C C C C, is taken, and we get as a result the lower curve $D D D D$, which is a compound of the two other curves, and as the result it will be seen that where we got the curve c, giving us a clifference of nearly five minutes, and the curve $\mathrm{B}$, giving a difference of about nine minutes in the same direction, we have a very great departure between the motions of the real and mean suns. Above and below the datum line, which is marked zero, we have 5, ro, and 15, which represent the difference in minutes at which the southings of our real and fictitious suns really take place. Early in the month of February we have a difference of very nearly fifteen minutes between the two suns, and it is at this time of the year of course that the sun dial is most in error. At other points where the effect of curve $B$ is to cause a great difference, the effect of curve $\mathrm{C}$ will be to minimise that difference, and so in the compound curve $D$ the difference is very slight. About the middle of June we get them together, then towards the end of July we get another separation, and about November I we come

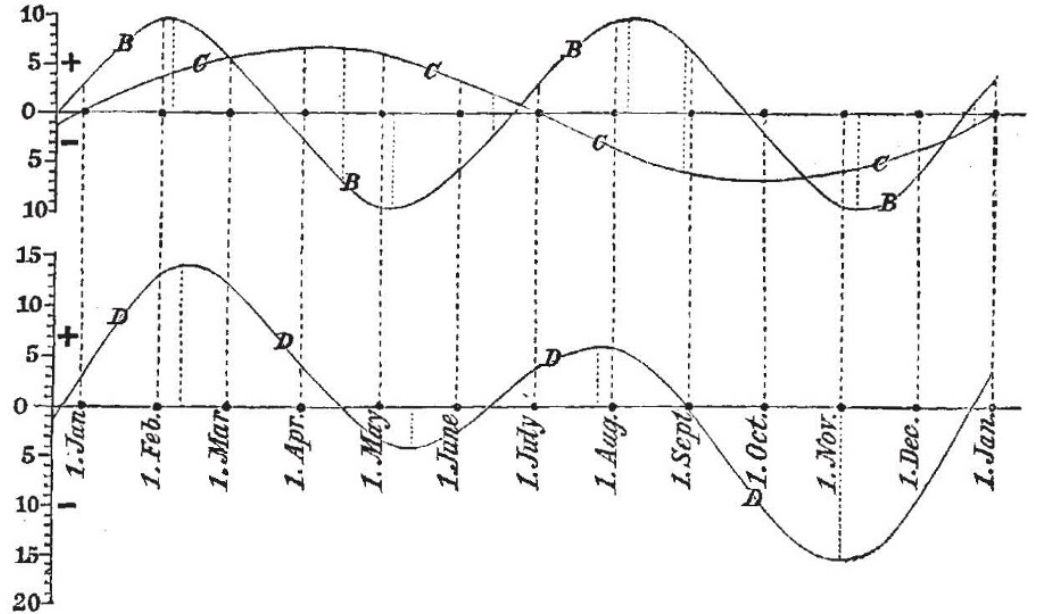

FIG. 52.-Diagram showing how the equation of time (curve D D D D) results from the combination of curve B B B B representing the vairation due to the obliquity of the ecliptic, and curve $\mathrm{C} \mathrm{CCC}$ representing the difference between the mean and true suns.

to another difference even greater than that in February. In this way a correction has been introduced, which is known as the "equation of time," and this added to the motion of the true sun, or added to that of our imaginary sun, brings them together, and by this means the mean sun is kept as nearly as possible to the average position of the true sun throughout the year. Another diagram (Fig. 53) will enable us to understand some of the considerations which have brought this about. Let $F$ represent the position of the sun in one of the foci of the ellipse, $\mathrm{P} e \mathrm{~A}$, round which the earth is supposed to be travelling. Now while we have the real radius vector going from $\mathrm{P}$ to $e$, with its unequal motion along the orbit, we have a fictitious radius vector going with absolute constancy along the circle. We get what is called the true anomaly in the angle P F $e$, and the mean anomaly in P F $e^{\prime}$, and the difference $e \mathrm{~F} e^{\prime}$ is called the equation of the centre. This equation helps us to determine those curves to which reference has been made, and the chief object in calling attention to this diagram is to explain the meaning of the term anomalistic year, which it will be necessary to introduce presently. It has already been said that it is imperative, if we are to gain any advantage from it, that real sun-time and apparent sun-time should never be widcly separated, because if so we might have 
contented ourselves simply with sidereal time, which would have at least the advantage of being constant, so that it is most necessary if any benefit is to be derived from this mean sun of ours that it should not differ very much from the true sun. The longitude of our mean sun is therefore made equal to the mean longitude of the true sun. This having been premised, the terms "mean time" and "mean noon" will now be clear without any explanation. "Greenwich mean time" of course means time referred to the meridian of Greenwich.

We thus finally discard our sidereal time, and replace it by mean solar time so arranged that the maximum departure of this from true solar time shall be fifteen minutes in the month of February and fiftecn minutes at the beginning of November. We have seen that the sidereal day is shorter than a solar day, and that consequently the hours, minutes, and seconds which

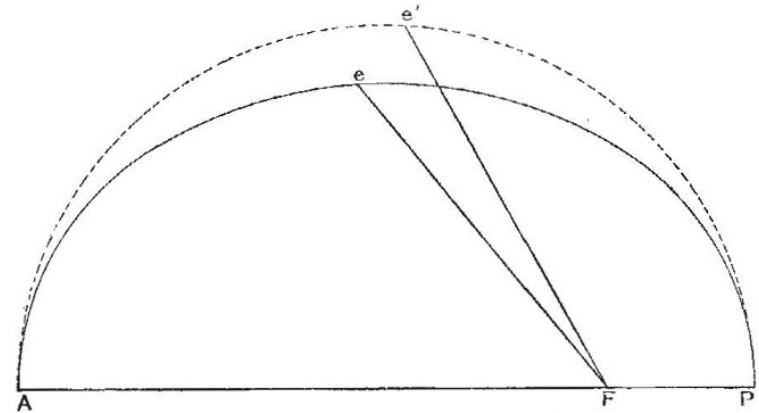

FIG. 53.-Diagram explaining mean anomaly and true anomaly.

make up the sidereal day must be shorter than those which form the solar day. The relation between the seconds of solar and sidercal time may be thus shown.

One sidereal second $={ }^{\circ} 9973$ of a mean-time second.

One mean-time sccond $=1 \cdot 0027$ of a sidereal second.

We have now got the results of the earth's revolution combined with its rotation, so far as day and night, considered in their more general aspects, are concerned; but we have not done with day and night yet. When we were considering the question of the inclination of the earth's axis, we went so far as to say that it was inclined $23^{\frac{1}{2}}{ }^{\circ}$ to the plane of the ecliptic, and that it always remained practically parallel to itself. Now suppose we arrange four globes in a circle, to represent the earth in different parts of its orbit, and we have in the centre an electric lamp to represent the sun. Then, if the earth's axis is thus inclined to its path round the sun, and always remains parallel to itself, it will be seen that at one position the north pole will be all in the light of the electric lamp (which represents the sun) during the entire reyolution of the earth on its axis.
At the point opposite this the reverse happens, for during the entire rotation of the earth the north pole will be in the dark. At the two remaining points the pole will be just on the boundary of light and darkness. We need not consider the case of the south pole; there exactly the reverse will happen to what occurs at the north pole--when the north pole is always in the light, the south pole will be always in the dark, and vice versâ, as may be seen by looking at the globes. Now it should be clear that the fact of the earth's axis being inclined to its path causes different lengths of day and night throughout the year. It is simply that, and nothing else. At the poles, which, as we have seen, are sometimes entirely in the light and sometimes altogether in the dark, there will be six months of this light and six months of darkness. At the equator it will be readily understood the days and nights will be of twelve hours' duration at whatever part of her orbit the earth may be. If you take those positions of the earth where the boundary of light and darkness passes through both the poles, it is perfectly clear that the days and nights are of equal length all over the world, and a line drawn from those points through the sun is therefore called the "line of equinoxes." These points are respectively at the ascending and descending nodes of the orbit. The two other points where the North Pole is most in the light or in the dark during the whole of a rotation are known as the solstices, because it is at these times that the sun for some days appears to attain the same height at noon.

To sum up then, it will be seen that the earth's rotation and the earth's revolution, in conjunction with the important fact of the non-coincidence of the planes in which they take place, give us not only our days and our nights, but cause the lengths of them in different latitudes to vary throughout the year. We have in this inclination of these planes to each other, too, the cause of the seasons, because when the northern hemisphere of the earth has been for a long time in that position with the sun longest above the horizon, the temperature will be very different to what it is when the earth is in the other position. In the former position we have summer in the northern hemispherc, in the latter winter. The conditions of life at two such points in the orbit will be vastly different. At the equator, where the days and nights are always of equal length, the course of nature will be very uniform. As the cquator is receded from and the poles are approached, this uniformity begins to disappear until, as has been said, at the pole six months of perpetual daylight alternate with six months when there is no sun.

But even now when we have gst our day and our year, we have not got all.

It must next be pointed out that, whilst the axis of the earth may be said to remain practically parallel to itself, yet that it does not absolutely remain so.

As a result of this and of the earth's movement round the sun, we get a very important outcome. Although the consideration of the dimensions of the earth has scarcely come within our sub-

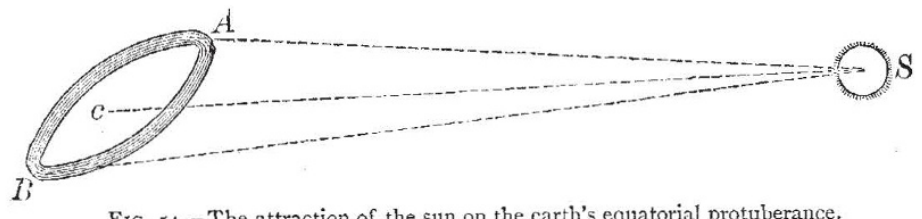

FIG. 54.-The attraction of the sun on the carth's equatorial protuberance.

ject, yet the earth's rotation may be used to bring in the dimensions of the body on which we dwell in just the same way as the velocity of light was used to refer to the dimensions of its orbit.

We need not, however, consider the question in detail, but we may state that the earth is a globe of something like 8000 miles in diameter, the equatorial diameter being longer than the diameter from pole to pole by some twenty-six miles, so that we have, as it were, round the equator a ring of matter some thirteen miles thick and eight thousand in diameter. Now this ring of matter, this equatorial protuberance, is presented to the sun at an angle to the line joining the centres of the sun and earth, as shown in Fig. 54, and the sun's attraction upon it can be resolved into two forces, one parallel to the line joining the centres of the sun and earth, and the other at right angles to this direction; and if we consider what will be the effect of this latter force upon such a ring, we can easily understand that it will result in an alteration of the inclination of the ring. In an arrangement for showing the effect of this attraction, the ring of matter on which the sun acts may be represented by an iron ring attached to a spinning top, and the resolved portion of the sun's pull may be imitated by the attraction of a magnet held in a nearly vertical position. As the ring rotates, the attraction of the magnet draws the ring out of the horizontal, and the poles revolves in a circle. This is what takes place with the earth's axis; hence it is not true to say that it always remains parallel to itself. This revolution is always slowly going on, being completed in a period of about 25,000 of our years. In consequence of this motion, what happens is this: the line of equinoxes which is at right angles to the line of solstices is constantly changing its position along the earth's orbit, producing what is called the precession of 
the equinoxes. We have to consider, therefore, not me:ely the sidereal year, the time between which the earth is at one point with reference to the sun and a star, and the time when it is at that same point again; we have not mercly to consider the fact that this line of solstices, with its conjoined line of equinoxes, varies with regard to what is called the apse line, that is, the line joining the perihelion and aphelion points of the orbit, or the axis-major of the ellipse--but we get from this another year which is called the tropical year, which, like our mean time, is the one most used, because it brings the year into relation with our seasons. Now that we have got our mean time and know exactly how and why we have got it, we may express the sidereal year in mean time, and say that it consists of 365.256 solar days. The tropical year - the time which elapses between two successive passages through the vernal equinox-is shorter than the sidereal one, owing to the precession along the orbit of the equinoctial points, and consist of $365^{\circ} 242$ mean solar days, and the difference between the lengths of this and the sidereal year will of course give the annual amount of precession which takes place. Anomalistic year is the term applied to the period which elapses between two successive passages through the perihelion or aphelion points of the orbit ; and as these points have a forward motion along the orbit, this year is longer than the sidereal one, being $365^{\circ} 259$ mean solar days.

We may give the exact lengths of these years in days, hours, minutes, and seconds as follows :-

\begin{tabular}{llllllll} 
& & \multicolumn{4}{c}{ Mean solar time } \\
d. & h. m. & s. \\
Mean sidereal year $\ldots$ & $\ldots$ & $\ldots$ & 365 & 6 & 9 & $9 \cdot 6$ \\
Mean tropical year ... & $\ldots$ & $\ldots$ & 365 & 5 & 48 & $46 \cdot 054440$ \\
Mean anomalistic year & $\ldots$ & $\ldots$ & 365 & 6 & $\mathbf{1 3}$ & $49 \cdot 3$
\end{tabular}

The Movcments of the Earth are so important to us, and so interesting in themselves, that it is not possible in six lectures to exhaust all that may be said about them or learned from them. I trust however that I have left no point of the first importance untouched. The moral of these lectures is that astronomy has appealed to physics, and has not appealed in vain, for the demonstration of the physical reality of the movements in question.

J. NORMAN LOCKYER

\section{THE FRENCH ASSOCIATION FOR THE PROGRESS OF SCIENCE}

THIS Association began its meetings at Blois on September 3 . The financial situation of the Association is very prosperous indeed; the capital has amounted to 20,0002 . ; but the sum spent in scientific researches amounts to only $300 \%$.

The President of the Association for this year is M. Bouquet de la Grye, and his inaugural address consisted of a sketch of the history of oceanic hydrography. He dealt with the difficulty of the determinations made on the bottom of the sea, and insisted on a new idea of his own. He believes that the level of the sea presents considerable variations owing to the quantity of salt contained in the water. He says that the level of the Mediter ranean ought not to be so high as the level of the ocean owing to the greater quantity of salt and consequently of density. A diminution of temperature produces the same effects as enlarging the density; so an increase of the temperature of the German Ocean would produce a flood on the Belgian, Dutch, German, and French coasts, and bring the sea to Paris.

Dr. Grimaux, a pupil of the late M. Wurtz, delivered a speech on the illustrions Academicians who have died during the past year, among whom Dumas and Wurtz have unquestionably the foremost place.

It is probable that this year the long-hoped-for fusion with the Association Scientifique de France, established by Leverrier, and presided over by Milne-Edwards, will take place, and the two Associations amalgamated in one will take a new start.

One of the principal objects of the present sitting has been the examination of the Thenay geological strata, wherc Abbé Bourgeois thinks he has discovered Tcrtiary man. The principal French geologists have arrived in Blois for the excursions. There arc very few foreigners at the meeting.

TRAINING IN NAVAL ARCHITECTURE ${ }^{1}$

$\mathrm{A}^{\mathrm{T}}$ Govan, the great shipbuilding suburb of Glasgow, on the 4th inst., Prof. F. Elgar, of Glasgow University, addressed the students attending the Science and Art Classes upon the I Communicated by Prof. Elgar. above subject. In the course of his address Prof. EIgar said :-

"All of the students wh) attend the classes in naval architecture and engineering here are probably much better acquainted with the practical and experimental aspects of the work they are engaged in than they are with the science which underlies it; and their present object is the very vital and praiseworthy one of acquiring such scientific and technical knowledge as will enable them to apply sound principles to the performance of their work, and to assist them in dealing intelligently and successfully with the many difficult and novel questions which are constantly obstructing and puzzling them. There are no branches of mechanical art in which sound scientific knowledge is more essential and useful, or in which it is more necessary for theory and practice to go hand-in-hand together, than those of shipbuilding and engineering. A modern steamer is so complex a machine that no attempts to construct one without calling in the aid of science in some form-either directly or by copying what others have learned by it to do-could possibly end in anything but disastrous failure. Try to imagine a man who had never heard or read of any of the teachings of science attempting to construct a modern steamship-a man who did not know even of the proposition, said to have been demonstrated by Archimedes, that a floating body displaces a volume of water whose weight is equal to its own weight; and who was ignorant of the wonderful discoveries that have been made of the laws by which heat generated by the combustion of coal is converted into mechanical work through the agencies of the boiler and steam-engine. It only requires to state the matter in this bald form in order to show how hopelessly impossible and absurd such an attempt would be, and how vitally dependent shipbuilding and engineering are upon the past achievements and present teachings of science. On the other hand, the highest scientific talent the world has yet produced would be equally unable to arrive at a successful result simply by means of pure theory, however advanced, and by strict a priori methods. The course you are pursuing, and which I trust you will not depart from, is the one best calculated to insure for you the greatest success in your work and advancement in your various positions in life; and as in the daily practice of your profession you are perforce kept well abreast of the practical and experimental sides of your work I would now urge you, in the strongest manner possible, to cultivate most diligently and thoroughly a knowledge of the science and of those natural laws upon which the efficiency and success of your efforts depend. Whatever may be the character of your daily work, whether you are employed as engineers, draughtsmen, or mechanics-and I am very pleased to know that there are working mechanics who attend these classes, and who are among the most earnest, intelligent, and capable of the students-never rest satisfied till you know the meaning of all that you do and why you do it. Do not be content with merely learning methods of setting off work and performing calculations, or with copying processes you may have seen others employ. The man who merely does as he sees others do, without very well comprehending why they do it, and who works strictly by rule and line, looking to custom as the supreme authority, will never improve or advance himself, nor be of much real use in such times as these; nor will he find much interest in his work.

$$
\begin{aligned}
& \text { 'Custom, which all mankind to slavery brings, } \\
& \text { That dull excuse for doing silly things.' }
\end{aligned}
$$

Never look to custom as being a sufficient authority for anything, however respectable its antiquity may have made it; but be determined to understand for yourselves whether or not it is based upon sound and intelligible principles. Although we are now meeting under the auspices of the Young Men's Christian Association, I can safely recommend you to indulge freely a spirit of scepticism in this particular department of the Association's work. The region of science and of the pure intellect is not one in which you should be content to accept the mere authority of any one as final, or to test any question except by the standard of your? own reason. Do not be too eager to believe that anything you are told is correct until you are able to prove it for yoursclves, and till you no longer feel any ignorance or doubt in the matter. The necessity for combining wide scientific knowledge and sound theory with practical experience, in the carrying on of shipbuilding and enginecring operations, is daily becoming more and more pressing. If you tried to avoid it you could not. In this age of keen competition and rapid development, increasing demands are made upon all who are engaged in these important 